\title{
Dual Tree M-Band Wavelet Transform Model based Classification of Mammogram Images
}

\author{
C. Suba, PhD \\ Asst Professor in Computer Science, \\ Sri Lakshmi College of \\ Arts \& Science, \\ Tottiyam Post, \\ Chinnasalem Tk, Villupuram Dt.
}

\author{
S. Niranjanan, $\mathrm{PhD}$ \\ HOD \& Associate Professor in BCA, \\ Mohemed Sathak College of Arts \& Science, \\ Sholinaganalur,Chennai.
}

\begin{abstract}
Breast Cancer is the one of the leading causes of cancer mortality among women and second leading cause of cancer deaths worldwide after lung cancer. In the US, 1 in 8 women will be diagnosed with breast cancer in their lifetime. The proposed CAD system is implemented in MATLAB and the performance is analyzed in terms of classification accuracy. Experimental Results indicate that DTMBWT has emerged as a potentially dominant feature extraction technique for breast cancer diagnosis. The risk for breast cancer increases with age; most breast cancer are diagnosed after age 50 and about $95 \%$ of all breast cancers in the US occur in women 40 and older.
\end{abstract}

\section{Keywords}

MIAS images, Dual Tree M-Band Wavelet Transform and Support Vector Machine.

\section{INTRODUCTION}

Breast cancer is disease that is diagnosed among the women in the world $[1,2]$. Mammography is used as early screening tool for breast cancer\& Mammographic images are X-ray images of breast region [3]. To objective of this paper is to build a Computer Aided Diagnosis (CADx) model for performance of mammogram classification system using variance features is discussed. To classifying mammogram images into three stages; normal/abnormal in stage 1 , Microcalcifications(MCs)/Mass in stage 2, benign/malignant in stage 3 .

\section{RELATED WORK}

Various kinds of techniques have been proposed to improve the accuracy of breast cancer classification. The research in the areas of CAD systems for breast cancer detection and classification has been conducted in last few decades. R. Nithya and B. Santhi [4] using GLCM (Grey Level Cooccurrence Matrix) features. The highest classification accuracy obtained by this approach was $96 \%$ on mammograms from the DDSM. Aidarus M. Ibrahim, Baharum Baharudin, Abas Md Said and P.N. Hashimah et al.[5] proposed k-means clustering coupled with max pooling (k-means-max pooling) is compared with well known feature extraction method namely Bag-of-features combined two different classifier; K-Nearest Neighbor(KNN) and Support Vector Machine(SVM), achieved good performance with an average classification accuracy of $98.19 \%$, sensitivity of $97.09 \%$ and specificity of $99.35 \%$.Muhammad Talha used Programming (GP) based filter is proposed, while the fusion of Discrete Cosine Transform (DCT) and Discrete Wavelet Transform (DWT) features is used as an input to classifier. It achieve $96.97 \%$ accuracy, $98.39 \%$ sensitivity and $94.59 \%$ specificity for classifying mammograms into normal and abnormal (cancer) categories using SVM (Support Vector Machine) classifier and MIAS (Mammographic Institute Society Analysis) dataset.

\section{SAMPLE MAMMOGRAM IMAGES FROM MIAS DATABASE}

To evaluate the performance of the proposed mammogram classification system, experiments are carried out using Mammographic Image Analysis Society (MIAS) database images. It is an organization of UK research groups interested in the understanding of mammograms.

Each pixel of mammogram image in MIAS database has 8 bits depth and at a resolution of $50 \mu \mathrm{m} \times 50 \mu \mathrm{m}$ and clipped/padded; thus the size of every image is 1024 pixels $x$ 1024 pixels. This database consists of 322 Mediolateral oblique mammograms (161 pairs) with ground truth data for each one. Images are characterized according to density, class of abnormality, and the severity of the abnormality present. There are 112 dense, 104 glandular, and 106 fatty images. Among these images, there are 209 normal, and 113 suspicious images.

The class of abnormalities are microcalcifications(25 images), circumscribed masses (23 images), architectural distortion (19 images), miscellaneous as ill-defined masses (14 images), asymmetry (13 images) and speculated masses (19 images). All MCs and mass images are used for evaluating the proposed system. Fig.55 shows sample images from MIAS database.

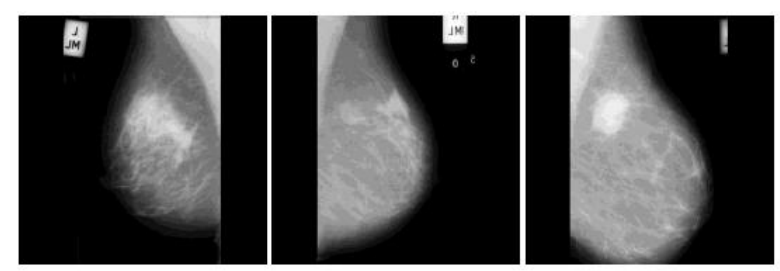

Figure 1: Sample Mammogram Images from MIAS Database

\section{METHODOLOGY}

Breast cancer is the most extensive cancerous pathology among women. It is one of the leading causes of cancer mortality among women. The decrease in breast cancer associated mortality may be attributable to several factors falling under the broad categories of treatment and detection. Both have been significantly advances over the last decades, 
due to the vast amount of research in the field. An overview of proposed method is presented in Figure 2.

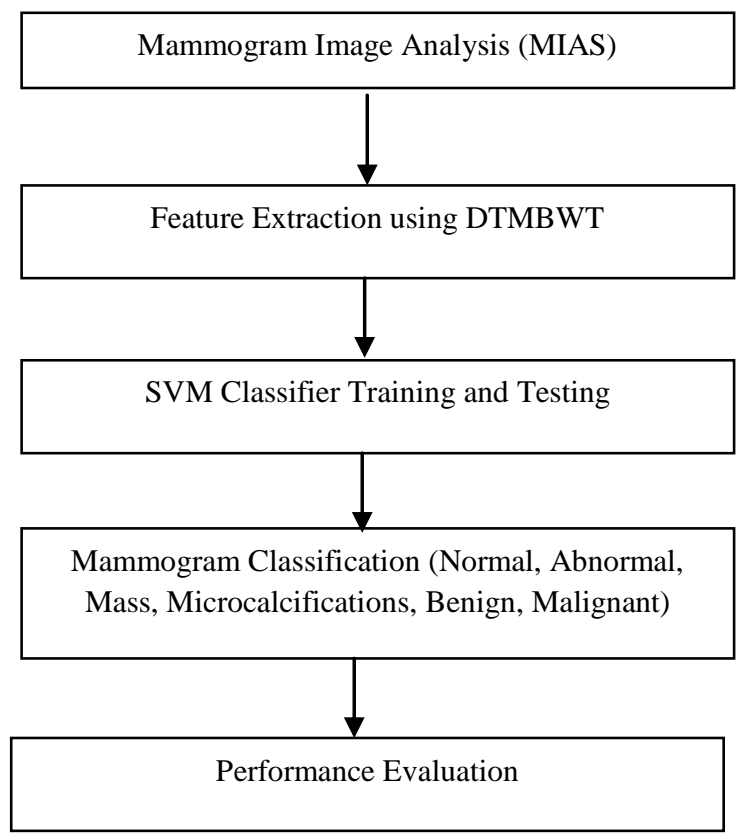

Figure 2: An Overview of Proposed Method

\subsection{Data Collection}

The most popular easily accessed databases and therefore the most commonly used databases are the Mammographic Image Analysis Society (MIAS) database and the Digital Database for Screening Mammography (DDSM). The Mammographic Image Analysis Society (MIAS) is an organization of UK research groups interested in the understanding of mammograms and has generated a database of digital mammograms. The database contains 322 digitized films and is available on $2.3 \mathrm{~GB}$ [6].

\subsection{Feature Extraction}

Feature extraction is very important part of pattern classification. In pattern recognition, feature extraction is considered as a critical process; owing to the features obtained from this process the efficacy of the classification process is directly influenced. Hence, feature with rich discriminative nature will assist the classification system effectively while the lack of such discriminative nature can slow down the classification process from accurate identification. Feature extraction using DTMBWT is performed by the following three computational modules. 1) ROI Selection 2) DTMBWT 3) Feature Computation $[7,8]$.

\subsection{Classification}

This is the final stage of the proposed mammogram classification system where the unknown mammogram is labeled into one of the predefined category of mammogram i.e. normal/abnormal, mass/MCs and abnormal severity into benign/malignant. A sequential classification stage that consists of three stages is designed for the classification of the given mammogram image using supervised learning based SVM classifier. In each stage, the performance of SVM classifier is analyzed using five kernel functions: linear, quadratic, polynomial, Radial Basis Function (RBF) and Multilayer Perception (MLP).

\section{PERFORMANCE MEASURE}

The performance of the prediction was evaluated in terms of classification accuracy, sensitivity, specificity, confusion matrix with Receiver Operating Characteristic (ROC) curve.

\subsection{Classification Accuracy}

The classifier performance is better if it has higher classification accuracy. It is defined by

$$
\text { Classificaionrate }(\%)=\frac{\text { Totalnumberof correctlyclassifiedimages }}{\text { Totalnumberof images }} \times 100
$$

\subsection{Sensitivity}

The sensitivity measure was first introduced in medical domain to ensure the test ability of the classifier [9]. It is calculated in the same way as the classification accuracy. However sensitivity regards only positive cases, for instance, it can be used to find patients with observed final diagnosis. In this study, sensitivity was computed as a number of true positive decisions over a number of actual positive cases. It can be represented as follows:

$$
\text { Sensitivity }=\frac{T P}{(T P+F N)}
$$

where $\mathrm{TP}=$ True Positive cases and FN $=$ False Negative cases.

\subsection{Specificity}

The specificity measure was also established in medical domain and computed in the same fashion as sensitivity[10]. The difference is that it deals only with negative cases, for example patients without observed final diagnosis. The specificity can be calculated as the number of true negative decisions over the number of actual negative cases. It can be represented as follows:

$$
\text { Specificit } y=\frac{T N}{(F P+T N)}
$$

where $\mathrm{TN}=$ True Negative cases and FP $=$ False Positive cases.

\subsection{Confusion Matrix}

For a two class problem, confusion matrix is constructed by using the classifier outcome with the actual one. Table 1 shows the confusion matrix.

Table 1 Confusion Matrix

\begin{tabular}{|c|c|c|}
\hline \multirow{2}{*}{ Test outcome } & \multicolumn{2}{|c|}{ Actual Class } \\
\cline { 2 - 3 } & Class 1 & Class 2 \\
\hline Class 1 & True Positive & False Positive \\
\hline Class 2 & False Negative & True Negative \\
\hline
\end{tabular}

In Table 1

$\checkmark \quad$ True positive: class 1 object is correctly classified as class 1 object

$\checkmark$ False Positive: class 2 object is incorrectly classified as class 1 object 
$\checkmark \quad$ True Negative: class 2 object is correctly classified as class 2 object

$\checkmark$ False Negative: class 1 object is incorrectly classified as class 2 object

\subsection{ROC Curve}

ROC curve is very useful for visualizing and analyzing the performance of a classifier. It shows the tradeoff between benefits (TP) and costs (FP). In addition, Area under the Curve (AUC) indicates the classifier performance.

\section{EXPERIMENT RESULT}

This section deals with experimental results of the evaluated mammogram classification system and their implications are discussed.

\subsection{First Stage Classification System}

The evaluated results of first stage classification system using variance features are illustrated in this section. Table 2 shows the classification accuracy of first stage classification.

Table 2: Classification Accuracy for Normal/Abnormal Classification System $\left(1^{\text {st }}\right.$ stage $)$ based on DTMBWT Variance Features and SVM Classifier

\begin{tabular}{|c|c|c|c|}
\hline \multirow{2}{*}{ Level } & \multicolumn{3}{|c|}{ Overall Classification Accuracy } \\
\cline { 2 - 4 } & Benign & Malignant & Average \\
\hline \multicolumn{4}{|c|}{ Polynomial } \\
\hline 1 & 96.74 & 49.29 & 73.01 \\
\hline 2 & 94.10 & 50.45 & 72.28 \\
\hline 3 & 90.92 & 54.93 & 72.92 \\
\hline 4 & 86.02 & 80.98 & 83.50 \\
\hline 5 & 78.29 & 73.82 & 76.05 \\
\hline 6 & 67.86 & 73.58 & 70.72 \\
\hline
\end{tabular}

The interpretations of Table 2 are as follows:

The maximum average classification accuracy while utilizing variance feature is $83.50 \%$. Among the five kernels used for normal and abnormal image classification, polynomial kernel achieves maximum classification accuracy while using $4^{\text {th }}$ level variance features of DTMBWT. Maximum average classification accuracy by linear, quadratic, RBF and MLP kernel is $75.76 \%, 72.30 \%, 74.55 \%, 70.00 \%$ respectively. It is also observed that all SVM kernels produce over $70 \%$ accuracy while using variance features.

In order to visualize the performance of the proposed $1^{\text {st }}$ stage classification using DTMBWT variance features and SVM classifier, confusion matrices and ROC plot are shown in Figure 3.

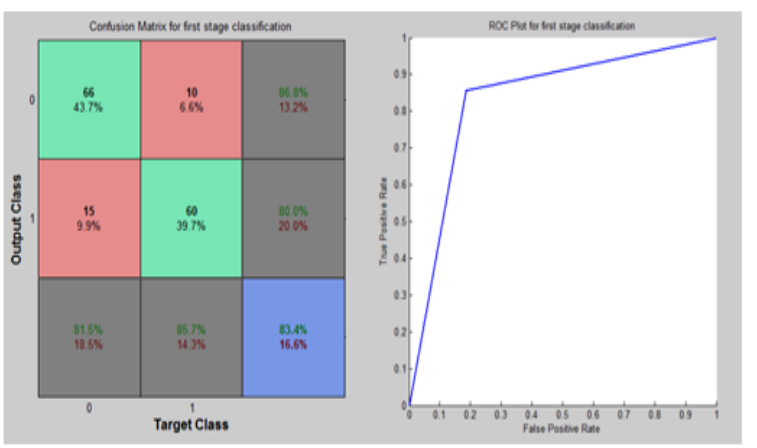

(a)

(b)

Figure 3: Performance of $1^{\text {st }}$ Stage Classification using DTMBWT Variance Features with Polynomial Kernel (a) Confusion matrix (b) ROC plot

It is observed from the Figure 3 that the 0.834 AUC is achieved while using Polynomial Kernel. It is observed from the Figure 2 that the sensitivity of the classification system is $81.5 \%$ with a specificity of $85.7 \%$. Figure 4 shows overall analysis of classification analysis of evaluated system in stage 1 .

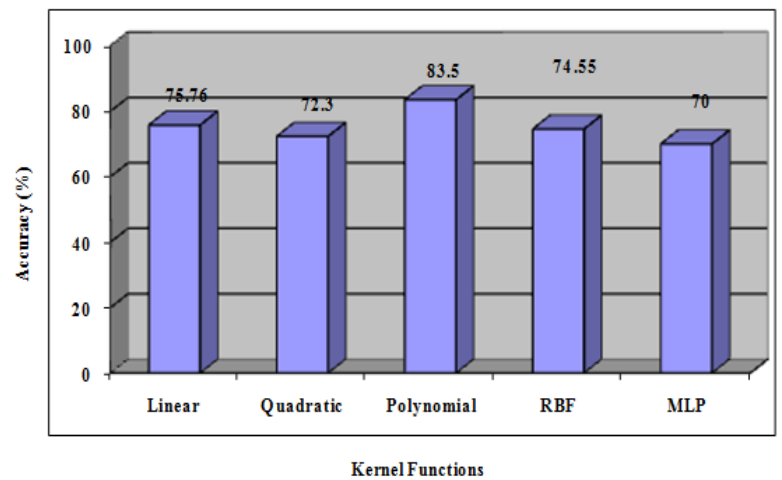

Figure: 4 Classification Accuracy vs. SVM Kernels for $1^{\text {s }}$ Stage Classification using DTMBWT Variance Features

\subsection{Second Stage Classification System}

The evaluated results of $2^{\text {nd }}$ stage classification system using variance features to classify the type of abnormality into mass or MCs are illustrated in this section. Table 3 shows the Classification Accuracy of $2^{\text {nd }}$ Stage Classification.

Table 3 Classification Accuracy for Mass/MCs Classification System ( ${ }^{\text {nd }}$ stage) based on DTMBWT Variance Features and SVM Classifier

\begin{tabular}{|c|c|c|c|}
\hline \multirow{2}{*}{ Level } & \multicolumn{3}{|c|}{ Overall Classification Accuracy } \\
\cline { 2 - 4 } & Benign & Malignant & Average \\
\hline \multicolumn{4}{|c|}{ Linear Kernel } \\
\hline 1 & 89.40 & 33.29 & 61.35 \\
\hline 2 & 85.04 & 45.30 & 65.17 \\
\hline 3 & 58.07 & 61.31 & 59.69 \\
\hline 4 & 81.12 & 79.99 & 80.56 \\
\hline 5 & 74.92 & 63.49 & 69.20 \\
\hline 6 & 62.70 & 62.97 & 62.83 \\
\hline
\end{tabular}




\section{Interpretations of Table 3 are as follows:}

The maximum average classification accuracy while utilizing variance feature for $2^{\text {nd }}$ stage classification is $80.56 \%$.Among the five kernels used for MCs/mass image classification, linear kernel achieves maximum classification accuracy while using $4^{\text {th }}$ level variance features of DTMBWT.Maximum average classification accuracy by, quadratic, polynomial, RBF and MLP kernel is $64.13 \%, 57.87 \%, 59.34 \%$ and $61.03 \%$ respectively. It is noted from the Figure 6 that the polynomial and RBF kernel achieves less than $60 \%$ classification accuracy.

In order to visualize the performance of the proposed $2^{\text {st }}$ stage classification using DTMBWT variance features and SVM classifier, confusion matrices and ROC plot are shown in Figure 5.

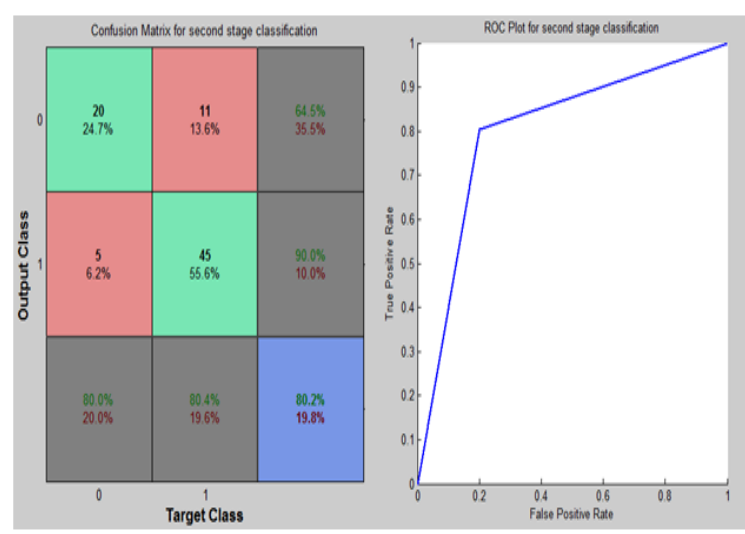

(a)

(b)

Figure 5: Performance of $2^{\text {nd }}$ stage Classification using DTMBWT Variance Features with Linear Kernel Confusion Matrix (b) ROC plot

In order to visualize the performance of the proposed $2^{\text {nd }}$ stage classification using DTMBWT variance features and SVM classifier, confusion matrices and ROC plot are shown in Figure 4. It is observed from the Figure 4 that the sensitivity of the proposed system is $80.0 \%$ with a specificity of $80.4 \%$. Figure 6 shows overall analysis of classification analysis of evaluated system in stage 2 .

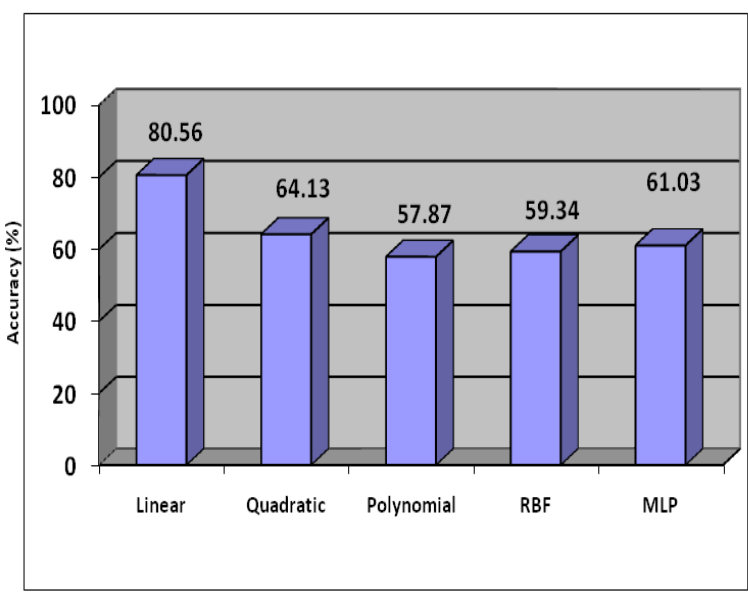

Kernel Functions

Figure 6: Classification Accuracy vs. SVM Kernels for $2^{\text {nd }}$ Stage Classification using DTMBWT Variance Features

\subsection{Third Stage Classification System}

In this stage, the abnormal severity of the classified mammogram that contains mass or MCs is again classified as benign or malignant. The output from the 2nd stage classification can be anyone of the abnormality; mass or MCs. As these two types of abnormalities have different characteristics, two different classification stages are designed for their severity classification into benign or malignant. The evaluated results of $3^{\text {rd }}$ stage classification system using DTMBWT variance features are illustrated in this section. Table 4 shows the classification accuracy of MCs severity classification

Table 4: Classification Accuracy for MCs Severity based on DTMBWT Variance Features and SVM Classifier

\begin{tabular}{|c|c|c|c|}
\hline \multirow{2}{*}{ Level } & \multicolumn{3}{|c|}{ Over all Classification Accuracy } \\
\cline { 2 - 4 } & Benign & Malignant & Average \\
\hline \multicolumn{4}{|c|}{ Polynomial } \\
\hline 1 & 38.67 & 69.71 & 54.19 \\
\hline 2 & 73.33 & 41.86 & 57.60 \\
\hline 3 & 88.50 & 64.38 & 76.44 \\
\hline 4 & 94.33 & 78.90 & 86.62 \\
\hline 5 & 92.17 & 51.21 & 71.69 \\
\hline 6 & 83.33 & 45.52 & 64.43 \\
\hline
\end{tabular}

The interpretations of Table $\mathbf{4}$ are as follows:

The maximum average classification accuracy while utilizing variance feature is $86.62 \%$ for the classification of MCs into benign/malignant. Among the five kernels used for MCs benign and malignant image classification, polynomial kernel achieves maximum classification accuracy while using $4^{\text {th }}$ level variance features of DTMBWT.Maximum average classification accuracy by linear, quadratic, RBF and MLP kernel is $70.77 \%, 69.15 \%, 67.26 \%$ and $62.98 \%$ respectively.

In order to visualize the performance of the proposed $3^{\text {rd }}$ stage MCs classification using DTMBWT variance features and SVM classifier, confusion matrices and ROC plot are shown in Figure 7. It is observed from the Figures 7 that the 0.840 AUC is achieved while using polynomial kernel. It is observed from the Figure 6 that the sensitivity of the proposed system is $76.9 \%$ with a specificity of $91.7 \%$. Figure 7 shows overall analysis of classification analysis of evaluated system in stage 3 MCs Classification.

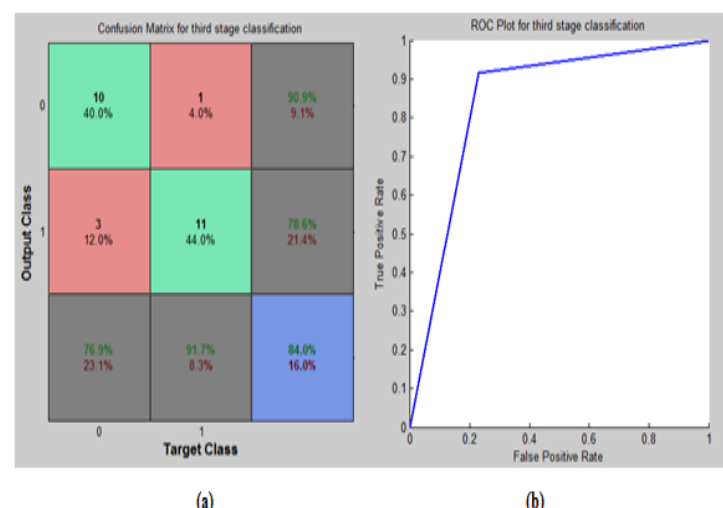

Figure 7: Performance of 3rd Stage MCs Classification using DTMBWT Variance Features with Polynomial Kernel (a) Confusion Matrix (b)ROC plot 


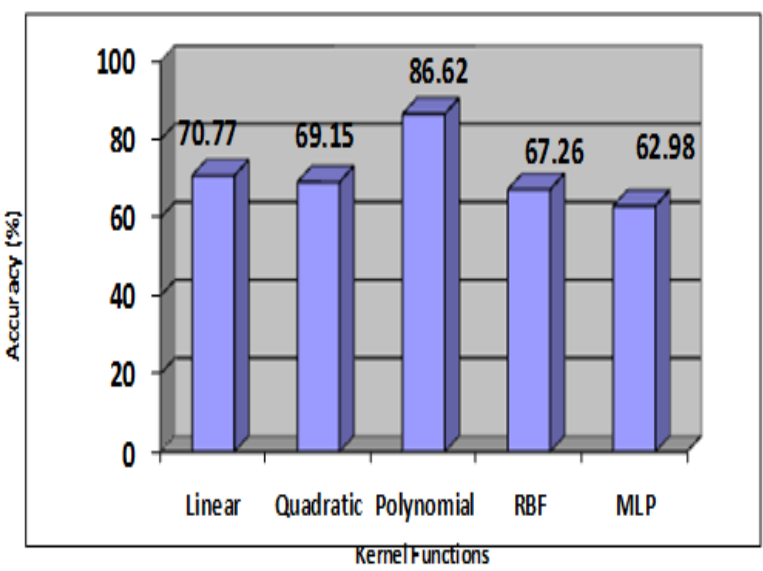

Figure 8: Classification Accuracy Vs. SVM Kernels for 3rd Stage MCs Classification using DTMBWT Variance Features

The evaluated results of 3 rd stage classification system using DTMBWT variance features are illustrated in this section. Table 5 shows the classification accuracy of Mass severity classification.

Table 5: Classification Accuracy for Mass Severity Based on DTMBWT Variance Features and SVM Classifier

\begin{tabular}{|c|c|c|c|}
\hline \multirow{2}{*}{ Level } & \multicolumn{3}{|c|}{ Overall Classification Accuracy } \\
\cline { 2 - 4 } & Benign & Malignant & Average \\
\hline \multicolumn{4}{|c|}{ MLP } \\
\hline 1 & 24.08 & 85.65 & 54.87 \\
\hline 2 & 46.77 & 74.35 & 60.56 \\
\hline 3 & 66.73 & 100.00 & 83.37 \\
\hline 4 & 55.83 & 88.65 & 72.24 \\
\hline 5 & 58.61 & 69.63 & 64.12 \\
\hline 6 & 55.61 & 71.77 & 63.69 \\
\hline
\end{tabular}

The interpretations of results of Table 5 are as follows:

The maximum average classification accuracy while utilizing variance feature is $83.37 \%$.Among the five kernels used for mass severity classification, MLP kernel achieves maximum classification accuracy while using $3^{\text {rd }}$ level variance features of DTMBWT.Maximum average classification accuracy by linear, quadratic, polynomial and RBF kernel is $59.96 \%, 61.58 \%, 62.26 \%, 63.03 \%$ respectively. It is also observed that the quadratic kernel classifies the mass malignant images with $95.00 \%$ accuracy while MLP kernel classifies with $100 \%$ accuracy.

In order to visualize the performance of the proposed 3rd stage mass classification using DTMBWT variance features and SVM classifier, confusion matrices and ROC plot are shown in Figure 9.

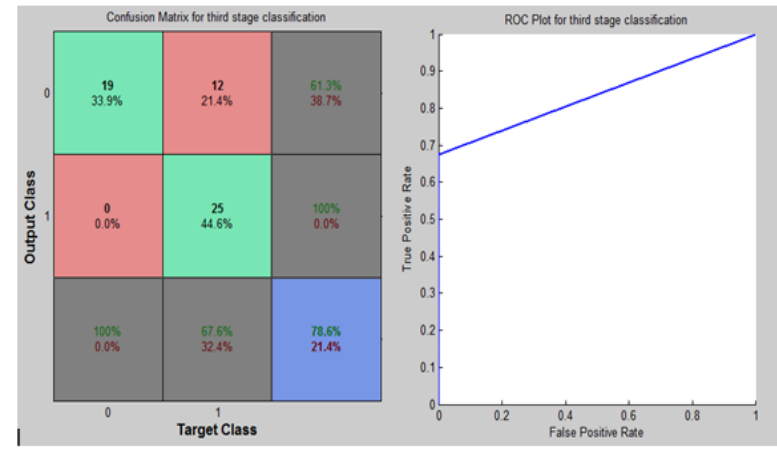

(a)

(b)

Figure 9: Performance of 3rd Stage Mass Classification using DTMBWT Variance Features with MLP Kernel (a) Confusion matrix (b) ROC plot

It is observed from the Figure 8 that the 0.786 AUC is achieved while using MLP kernel with the sensitivity of the proposed system is $100 \%$ with a specificity of $67.6 \%$. Figure 10 shows overall analysis of classification analysis of evaluated system in stage 3 Mass Classification.

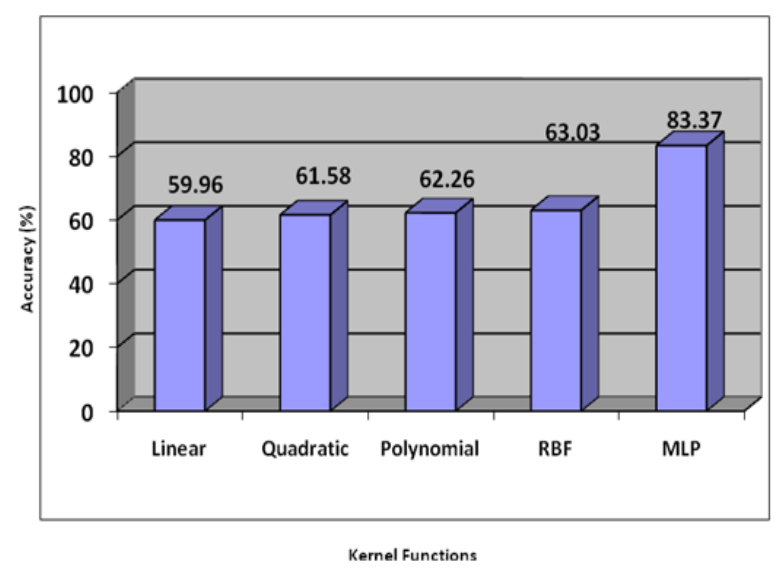

Figure 10: Classification Accuracy Vs. SVM Kernels for 3rd Stage Mass Classification using DTMBWT Variance Features

\section{CONCULSION}

The method employed in this paper has given better performance. The CAD system is developed for the classification of mammogram into 3 stages; Normal/Abnormal Classification in Stage 1, Mass/Micro calcifications (MCs) Classification in Stage 2,Benign/Malignant in Stage 3. This paper has investigated a classification of mammogram images using DTMBWT features. The maximum average classification accuracy rate is $83.50 \%$ for normal/ abnormal classification. Classification accuracy for Mass/MCs classification system in 2nd stage $80.56 \%$, Classifiaton accuracy for Mass Severity in 3rd stage $83.37 \%$. For future work, GLCM features combined with statistical moment features to improve statistical properties of DTMBWT coefficients, other textural features can be used to improve the results in the classification of digital mammogram images. For future work, Hybrid classifier or ensemble based classification can be used for further improvement. 


\section{REFERENCES}

[1] www.breastcancer.org

[2] Brijesh Verma and Ping Zhang."A novel neural-genetic algorithm to find the most significant combination of features in digital mammograms", Applied Soft Computing, no.7, pp.513-525, 2007.

[3] Marrocco, C., M. Molinara, C. D'Elia and F. Tortorella,2010. “A Computer Aided Detection System for Clustered microcalcifications. Artif. Intell. Med., 50:23-32. DOI: 10.1016/j.artmed.2010.04.007.

[4] R. Nithya and B. Santhi." Classification of Normal and Abnormal Patterns in Digital Mammograms for Diagnosis of Breast Cancer, International Journal of Computer Applications (0975 - 8887), Volume 28No.6, August 2011.

[5] Aidarus M. Ibrahim, Baharum Baharudin, Abas Md Said and P.N. Hashimah," Classification of Breast Tumor in Mammogram Images Using Unsupervised Feature
Learning”, Science Publications, Original Research Paper,2016.

[6] Mini MIAS Database Information available at: $\mathrm{http}: / /$ mammoimage.org/database..

[7] Caroline Chaux , Laurent Duva and Jean-Christophe Pesquet. "2D Dual-Tree M-Band Wavelet Decomposition".

[8] Selesnick.I.W."The Double-Density Dual-Tree DWT", IEEE Transaction on Signal Processing, 52(5), 13041314, 2004.

[9] Cheng. H. D. \& Cui. M. (2004). "Mass Lesion Detection with a Fuzzy Neural Network's”, 37(6), 1189-1200.

[10] Verma. B., McLeod. P, \& Klevansky. A. (2010). "Classification of Benign and Malignant Patterns in Digital Mammograms for the Diagnosis of Breast Cancer".Expert Systems with Applications, 37(4), 33443351. 